\title{
Directorio de expertos en el tratamiento de la información (EXIT). Análisis de uso
}

\author{
Por Enrique Orduña-Malea, Josep-Manuel Rodríguez-Gairín y Tomàs Baiget
}

Resumen: Descripción y características del Directorio EXIT (Expertos en el tratamiento de la información), puesto en marcha oficialmente en junio de 2005. A los dos años (julio de 2007) se ha evaluado y analizado su funcionamiento, implantación, visibilidad y aceptación por parte de la comunidad profesional de bibliotecarios, documentalistas, archiveros y especialistas en información a la que sirve, y en especial su uso. Técnicamente, EXIT está considerado un directorio estado-del-arte a nivel mundial, siendo además un genuino producto de la web 2.0 ya que son los propios interesados los que rellenan y mantienen al día sus fichas, bajo la supervisión de sus creadores-gestores y de un Comité Evaluador internacional.

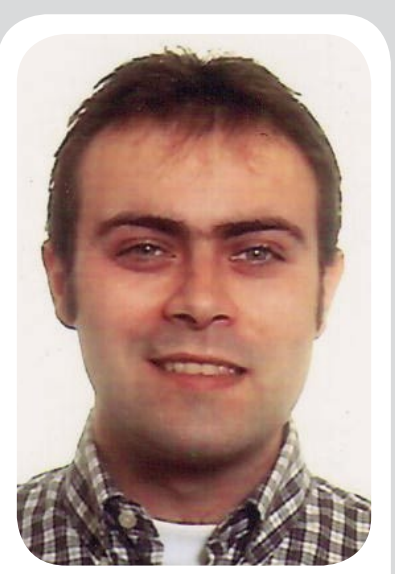

Enrique Orduña Malea es técnico especialista en imagen y sonido (especialidad imagen fílmica), ingeniero técnico en telecomunicaciones por la Escuela Politécnica Superior de Gandía y licenciado en documentación por la Univ. Politécnica de Valencia. Actualmente colabora en la Facultad de Informática de la UPV y en la biblioteca del estudio de arquitectura de Tomás Llavador.

Palabras clave: Directorio EXIT, Web 2.0, Bibliotecarios, Documentalistas, Archiveros, Especialistas en información, Directorios profesionales, Análisis de uso, Estadísticas

Title: Directory of Experts on Information Management (EXIT). Usage analysis

Abstract: The characteristics of the EXIT Directory (Experts in information management),

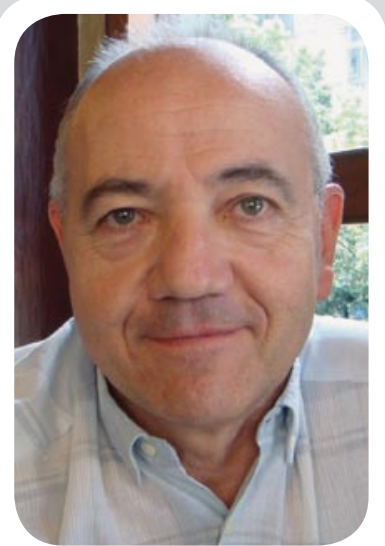

Tomàs Baiget, jefe de proyectos de información en la SDG de Difusión, del Institut d'Estadística de Catalunya (Idescat), donde también se encarga de la distribución-e de la revista SORT, es coordinador del Grupo ThinkEPI (el autor colectivo de los Anuarios ThinkEPI), editor español del repositorio E-LIS, y promotor del sistema de normalización de nombres de autor IraLIS, y profesor del Master Online de Documentación Digital del IDEC, Univ. Pompeu Fabra.

officially launched in June of 2005, are described. After two years online (July of 2007), its use was evaluated and analyzed, taking into account its operations, impact, visibility, and acceptance on the part of the professional community it serves: librarians, information scientists, archivists and information specialists. Technically, EXIT is considered a stateof-the-art directory and a genuine product of web 2.0, since those involved submit and update their own records, under the supervision and filtering of EXIT creators-managers and an international Evaluation Committee.

Keywords: EXIT Directory, Web 2.0, Librarians, Information scientists, Archivists, Record managers, Information specialists, Information managers, Professional directories, Usage analysis, Statistics

Orduña-Malea, Enrique; Rodríguez-Gairín, Josep-Manuel; Baiget, Tomàs. "Directorio de expertos en el tratamiento de la información (EXIT). Análisis de uso”. En: El profesional de la información, 2007, septiembre-octubre, v. 16, n. 5, pp. 497-509.

DOI: $10.3145 /$ epi.2007.sep.13

\section{Introducción}

EN LAS CIENCIAS DE LA GESTIÓN DE LA INFORMACIÓN Y DOCUMENTACIÓN, como en cualquier otra disciplina, suele aparecer con frecuencia la necesidad de disponer de información acerca de la propia profesión: metainformación la llamaríamos siguiendo la moda actual.

Algunos problemas usuales de la falta de la misma suelen ser:
- Imposibilidad de contactar con colegas con intereses profesionales comunes.

- Desconocimiento de trabajos o líneas de investigación afines.

- Desconocimiento de institu- 
ciones, tanto públicas como privadas, que puedan estar realizando actividades de nuestro interés.

Tradicionalmente, el problema de este tipo de información es que suele ser escasa, distribuida, obsoleta e inconsistente. Por tanto, existe una relativa necesidad en disponer de un sistema centralizado que proporcione información actualizada acerca de los profesionales e instituciones que se dedican, de una $u$ otra forma, al tratamiento intensivo de la información.

En este contexto aparecen los directorios como esa fuente de información que debe solucionar esta necesidad informativa. Un directorio se puede definir como (Carrizo, 2000) $)^{4}$ : "Una lista de personas u organizaciones, ordenada sistemática y alfabéticamente, figurando filiación, dirección, funciones y otros datos de interés para cada una de ellas". La publicación de directorios de organizaciones públicas y privadas ha sido habitual, pues suelen cumplir una función esencial para el sistema económico de un país: identificación de clientes y proveedores (Ferrer; Peset, 2007) ${ }^{5}$.

Pero una fuente de carácter personal, coherente y suficientemente completa, constituye también una herramienta eficaz de observación. Por ello, un análisis de la misma y de su uso debería proporcionar información interesante acerca de los profesionales y de la profesión en su conjunto.

Pese a ser fuentes de información comunes en otras ramas profesionales, los directorios no han proliferado mucho en nuestra disciplina (Membrillo; Jiménez, 1998) ${ }^{7}$. Como directorio online puede destacarse el realizado por Rafael Aleixandre $^{1}$. Por ello, tampoco existen muchos trabajos que traten de analizar y estudiar la información de los mismos. Recientemente cabe citar el trabajo realizado por el Grupo Scimago (Hassan-Montero; Herrero-Solana, 2007) ${ }^{6}$ donde se analiza la distribución y co-ocurrencia de los descriptores usados en el directorio EXIT.

Así, pues, los objetivos de este trabajo son:

- Presentar el Directorio $E X I T^{10}$, mostrando sus características, funciones y servicios en la actualidad, aprovechando el segundo aniversario de su puesta en marcha, que oficialmente fue en junio 2005 (funcionaba en período de pruebas desde enero de 2005).

- Analizar estadísticamente la cobertura y uso del directorio en el año 2006, para ayudar a establecer con ello un observatorio específico de la profesión.

El análisis se divide en cuatro apartados:

1. Distribución geográfica de los registrados, a fecha de 5 de junio de 2007.

2. Análisis del uso de los descriptores, tanto desde el punto de vista de los profesionales que se inscriben como de los usuarios que realizan búsquedas, con el objetivo de buscar posibles diferencias. Los datos se toman desde la puesta en marcha del directorio hasta el día 5 de julio de 2007.

3. Estudio y comparación de los datos de las visitas recibidas, tanto en número de clics como en visitantes, obtenidos durante el primer mes de funcionamiento y el último (junio 2007).

4. Finalmente, se analizan las consultas al directorio durante el último año. Para ello se tiene en cuenta la marca del navegador, las formas de acceder al directorio y el origen de las visitas, comparando en primer lugar los continentes y realizando posteriormente una descripción de España, Sudamérica y Estados Unidos.

\section{Material y métodos}

El análisis de uso del directorio se realizó mediante tres procedimientos:
- Directo a través de la información que proporciona la interfaz de búsqueda.

- Mediante Google Analytics, para capturar toda la información relativa al acceso de los usuarios durante el período de un año.

- Con el aplicativo de estadísticas Php-stats incorporado en EXIT, sobre el uso de descriptores en las búsquedas de los usuarios.

\section{http://php-stats.com/}

Todo el estudio se realizó durante la primera semana del mes de julio de 2007. Se hizo un recuento del número de registros almacenados, su procedencia geográfica así como los descriptores que caracterizan a cada profesional. Los datos obtenidos se volcaron a una tabla Excel, donde se analizaron.

La interfaz del directorio proporciona suficiente información como para no necesitar una captura especial de la misma.

El análisis indirecto se basó en Google Analytics. Con el objetivo de mostrar la evolución del directorio, se tuvo en consideración el período anual desde el 14 de junio de 2006 hasta el 14 de junio de 2007. El intervalo de inicio vino marcado por no poder disponer de datos anteriores a dicha fecha registrados en Google Analytics.

Mediante este análisis se obtuvieron los datos acerca del modo de acceso al directorio (navegador, tráfico, visitas en marzo y abril de 2007 y lugares de acceso).

Finalmente, para el análisis de los descriptores usados por los usuarios en las búsquedas, se utilizó la citada aplicación Php-stats, que proporciona datos desde el nacimiento del directorio, fechado a 1 de junio de 2005, así como las comparativas de clics y visitantes entre junio 2005 y 2007.

Para la recogida de datos, la elección de Google Analytics en lu- 
gar de Php-stats, que hubiese permitido un intervalo mayor -prácticamente desde el nacimiento-, vino motivada por las siguientes razones:

- Las funcionalidades de $G o$ ogle Analytics son muy superiores.

- No se podían filtrar adecuadamente los accesos realizados en las primeras semanas, todavía en fase de pruebas del directorio ( $1 \mathrm{de}$ junio a 12 de julio).

- No existían datos para el mes de junio de 2006 -debido a un problema puntual que se produjo ese mes-, cuando en Google sí aparecían.

\section{EI Directorio EXIT ${ }^{9}$}

\subsection{Introducción: misión y objetivos}

El nacimiento y crecimiento de todo sistema de información debe tener en cuenta desde un principio las condiciones físicas, sociales y culturales en las que está inmerso.

Cuando en 1992, con internet, empezó a proliferar el uso del correo electrónico, Tomàs Baiget hizo un primer listado de direcciones llamado DirIWE, Directorio de bibliotecarios y documentalistas de habla hispana. En las primeras versiones los datos se introducían manualmente, y la lista se mantenía como fichero ascii en el servidor de RedIris, la organización que provee los servicios de telecomunicaciones a la comunidad académica y científico-técnica española. En marzo de 1995 Josep-Manuel RodríguezGairín, entonces bibliotecario de la Univ. Politécnica de Cataluña, realizó nuevas versiones y mejoras: a la lista diriwe.txt añadió diriwe.dbf, para funcionar como base de datos en DBaseIII+, y diriwe.exe, que era un programa que se instalaba en el PC local y asistido por menús permitía buscar cualquier palabra del directorio (Fig. 1)2. Todo ello se podía bajar de un gopher de la UPC.

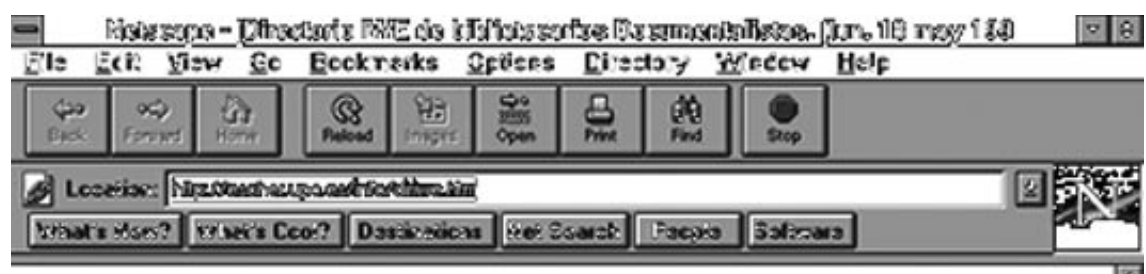

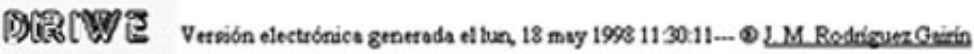

\section{Directoris [\%/E de Biblistecarios Documentalistas}

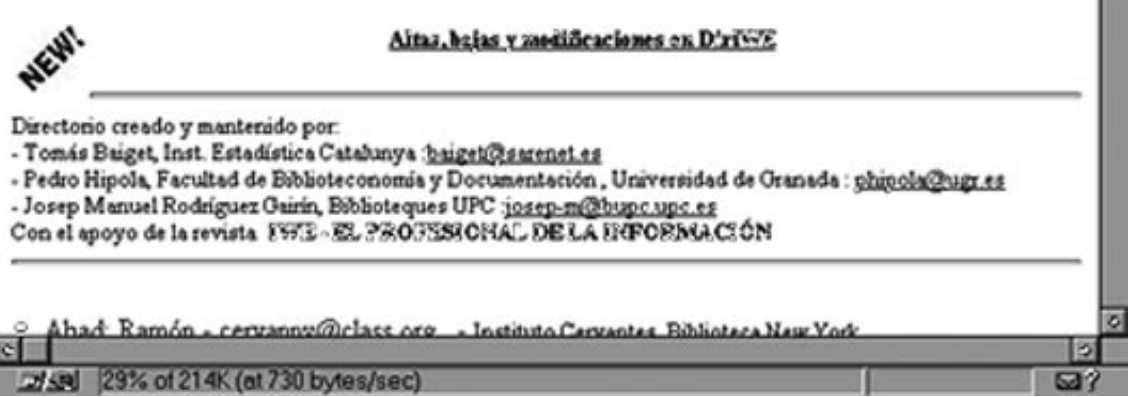

Fig. 1. Primera versión-e del antiguo DirIWE

Posteriormente, en agosto de 1997, se creó una versión gestionada a través de la web ${ }^{8}$ en la que los usuarios podían inscribirse autónomamente - y actualizarse mediante su password- . En esa época DirIWE tenía 1.100 nombres, aunque cada registro era de una sola línea: nombre, institución abreviada y dirección-e. Esta forma online estuvo activa hasta febrero de 2007 , cuando por un acuerdo entre los responsables de la UPC y de EXIT, las consultas a DirIWE empezaron a ser desviadas a EXIT.

Ni que decir tiene que la profesión está experimentando importantes cambios producidos por la inserción intensiva de las TICs en la creación, gestión y consumo de documentos. Esto ha propiciado la aparición de nuevos formatos y con ello de nuevas ramas en la profesión, como "arquitectura de la información", "bibliotecas digitales", "cibermetría" y un polémico etcétera.

En estos momentos, disponer de un directorio capaz de agrupar y estructurar información lo más completa posible acerca de las personas que se encuentran en la vanguardia de dichos campos es fundamental para la propia evolución de la disciplina y la difusión de la ciencia en general.

$E X I T^{10}$ es un directorio de uso gratuito, accesible online, que nació oficialmente en junio de 2005 , después de cinco meses de pruebas y mejoras. En su elaboración y mantenimiento participan JosepManuel Rodríguez-Gairín (desarrollo técnico y gestión), Tomás Baiget (coordinación y promoción) y Jorge Franganillo (diseño e interacción).

Sus dos propósitos principales son:

- Centralización y estructuración de información sobre la profesión. Un directorio ayuda a vertebrar, a dar consistencia y coherencia a un colectivo, permitiendo ver quién está y quién no, y con qué características figura en él.

- El establecimiento de una puerta de entrada a la ciencia mediante la combinación del directorio con otros elementos para formar un sistema de información más completo. EXIT ofrece valiosas interrelaciones al poder agrupar a los miembros por especialidades, por ciudades o países, etc. 
Bajo estas dos importantes misiones, los objetivos marcados para poder cumplirlos se muestran a continuación:

- Conseguir una masa crítica de profesionales que hagan del directorio un referente de información internacional.

- Ofrecer información consistente y actualizada.

- Ofrecer información difícil o imposible de localizar a través de buscadores (aunque a los pocos días los buscadores permiten encontrar a su vez fácilmente los registros de EXIT, pues los sitúan en primer lugar o muy altos en el ranking de resultados, al preguntar por cualquier miembro).

- Permitir a los profesionales ponerse en contacto entre ellos.

- Facilitar la localización de profesionales adecuados para su participación en eventos, congresos, conferencias, etc., o para ofrecerles un mejor puesto de trabajo.

- Agrupar a los mejores profesionales de la información (archiveros, bibliotecarios, documentalistas, etc.), creando sentido de comunidad profesional a nivel universal.

\subsection{Funcionamiento y carac- terísticas}

Las personas interesadas en formar parte de EXIT deben realizar una inscripción a través de un formulario. La solicitud es evaluada por un comité formado por 17 expertos del más alto nivel. Para poder evaluar positivamente una solicitud, ésta debe ser aprobada al menos por dos miembros del comité; de esta forma se asegura que todos los profesionales inscritos hayan alcanzado un mínimo de méritos en la profesión, aumentando con ello la calidad del directorio. Esa última característica de selección de los miembros es uno de sus valores añadidos más interesantes, por lo que es usado a la hora de buscar ponentes, conferenciantes, profeso- res o socios de proyectos, en cada una de las 48 especialidades en que están clasificados.

Uno de los principales factores que condicionan el crecimiento de un directorio es la reticencia de las personas a incluir información considerada personal, así como la inclusión de un currículum y una fotografía en primer plano (elemento éste que se considera indispensable para una correcta identificación, que al fin y al cabo es lo que se persigue). Los gestores de EXIT tienen que dedicar un tiempo a informar a los colegas que en su CV no hace falta que figuren datos sensibles tales como fecha de nacimiento, números de identidad, seguridad social, domicilio particular, etc. Se piden datos estrictamente profesionales.

Las características técnicas que ofrece son, fundamentalmente, las siguientes:

- Mecanismos que impiden la captura de las direcciones de correo-e por parte de los robots (el signo@ es una imagen).

- Estandarización de entidades.

- Inserción opcional de currículo en pdf, pensado especialmente para las personas que todavía no tienen web personal.

- Enlace automático a las obras depositadas por el autor en el repositorio E-LIS.

- Posibilidad de edición de la ecuación de búsqueda en Google Scholar y en Google, Yahoo y MSN, con el fin de optimizarla a cada persona.

- Obtención de una medida de la popularidad de cada uno de los URLs anotados en las fichas, incluyendo un mapa TouchGraphicsGoogle.

- Comprobación automática en tiempo real de la validez de todas las direcciones mostradas en la ficha.
- Conversión inmediata de las fichas a los formatos MADS (metadata authority description schema) y $v$ Card.

\subsection{Diseño}

El diseño de todo sistema de información debe atenerse a unas prioridades y a unas limitaciones o restricciones. En el caso de EXIT, el criterio principal ha sido obtener la máxima eficacia funcional y la máxima exactitud. Por ello siempre han sido una prioridad los siguientes parámetros:

- Centralización de la edición de la información para que sea coherente y normalizada según unos criterios formales predefinidos.

- Calidad de la información (antes de aprobar las fichas se comprueba la veracidad de la información más importante de cada solicitante). Si es necesario se recurre al Comité Evaluador.

- Usabilidad, accesibilidad y diseño.

- Privacidad de la información, un compromiso explícita y públicamente asumido por los gestores.

Como el directorio debe ser una puerta de entrada a la información de un conjunto de personas, clasificadas en base a diferentes criterios, la elección de un sistema de fichas resulta adecuada. Cada ficha dispone la información estructurada en campos claramente diferenciados para todos los inscritos.

El diseño intenta ser atractivo visualmente y no sobrecargar la interfaz de información.

Desde todas las páginas se ofrecen 4 opciones de color de la interfaz, así como poder cambiar el idioma. La interfaz está disponible en inglés, español e italiano y el sistema selecciona automáticamente cada una en función del idioma configurado en las opciones del navegador del usuario. En caso de otros idiomas sale el inglés por defecto. 
Para los usuarios más avanzados, la conversión de las fichas a otros formatos (MADS y vCARD, como se ha dicho) permite su reutilización y transferencia de forma estructurada, y favorece su recuperación.

MADS (Metadata Authority Description Schema) es un estándar impulsado por la library of Congress para intercambiar registros de autoridades que permite establecer relaciones recíprocas con catálogos bibliotecarios. VCard es el estándar para el intercambio de información personal usado en agendas electrónicas y firmas en gestores de correo como Outlook o Mozilla Thunderbird.

En la siguiente figura se muestra la interfaz principal del directorio:

EXIT está alojado en servidores de la empresa Kronosdoc, en Barcelona.

\subsection{Adquisición e introduc- ción de datos}

Los datos se introducen en el sistema a través de un formulario al que se puede enlazar desde la página de inicio. Los campos que se deben introducir son los siguientes:

- Nombre $(*)$

- Apellidos (*)

- Institución

- País

- Ciudad

- Provincia (este campo se activa solamente cuando el usuario elige España como país)

- Dirección

- Código postal

- Teléfono/móvil/fax

- E-mail institucional y personal

- Web institucional y personal

- CV (pdf) (*)

- Fotografía (jpg o gif) $\left(^{*}\right)$

\section{exit manas}

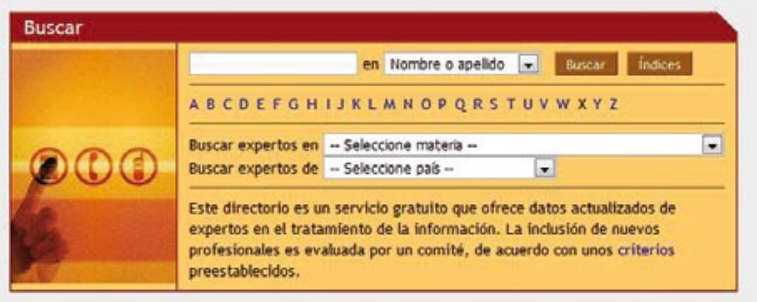

Fig. 2. Interfaz del directorio EXIT

- Descriptores (a elegir como máximo 5 de una lista, o introducir 1 más en texto libre)

Los campos marcados con un asterisco $(*)$ son obligatorios para atender cada petición. La inclusión de datos en los campos restantes es voluntaria.

Una vez el usuario rellena el formulario y envía la solicitud, si ésta es aceptada, se genera automáticamente la ficha correspondiente, quedando visible en el directorio.

Al tratar información personal y sensible, la protección de la información en el directorio es fundamental. Desde la portada se ofrece un enlace a la página de la política de privacidad que rige el directorio.

Acorde con lo establecido por la Ley Orgánica 15/1999, de 13 de diciembre, de Protección de Datos de Carácter Personal, EXIT informa al usuario de la existencia de un fichero automatizado de datos de carácter personal. Los datos de los usuarios, registrados de forma libre y voluntaria a través del formulario, son recabados por EXIT con la finalidad de elaborar el directorio.

Así mismo, los gestores de EXIT declaran no hacer ni permitir spam y únicamente trata los datos que el usuario aporta mediante los formularios habilitados en este sitio web o mensajes electrónicos. Además, pone todos los medios técnicos a su alcance para evitar que los miembros del directorio puedan recibir cualquier tipo de correo indeseado.

\subsection{Acceso a la información}

La página inicial ofrece tres tipos de búsqueda:

1) Búsqueda libre:

- Nombre y/o apellidos

- Institución

- Dirección

- Ciudad

- Provincia

- Correo

- Descriptor personal

2) Índice alfabético:

Adicionalmente se puede buscar a través de un índice alfabético (apellidos de los inscritos).

3) Descriptores controlados

- De materia

- De país de procedencia

\subsection{Ventajas y usos de EXIT}

- Estructuración de la información de contacto de profesionales.

- Acceso a un tipo de información no siempre disponible o fácil de encontrar.

- Posibilidad de detección de colegios invisibles, focos de investigación y estudios relativos a la distribución de científicos de una determinada área o materia.

- Localización rápida y eficaz de científicos, autores y colegas en general.

- Ayuda al conocimiento de la evolución científica de los autores más importantes del sector y de la 


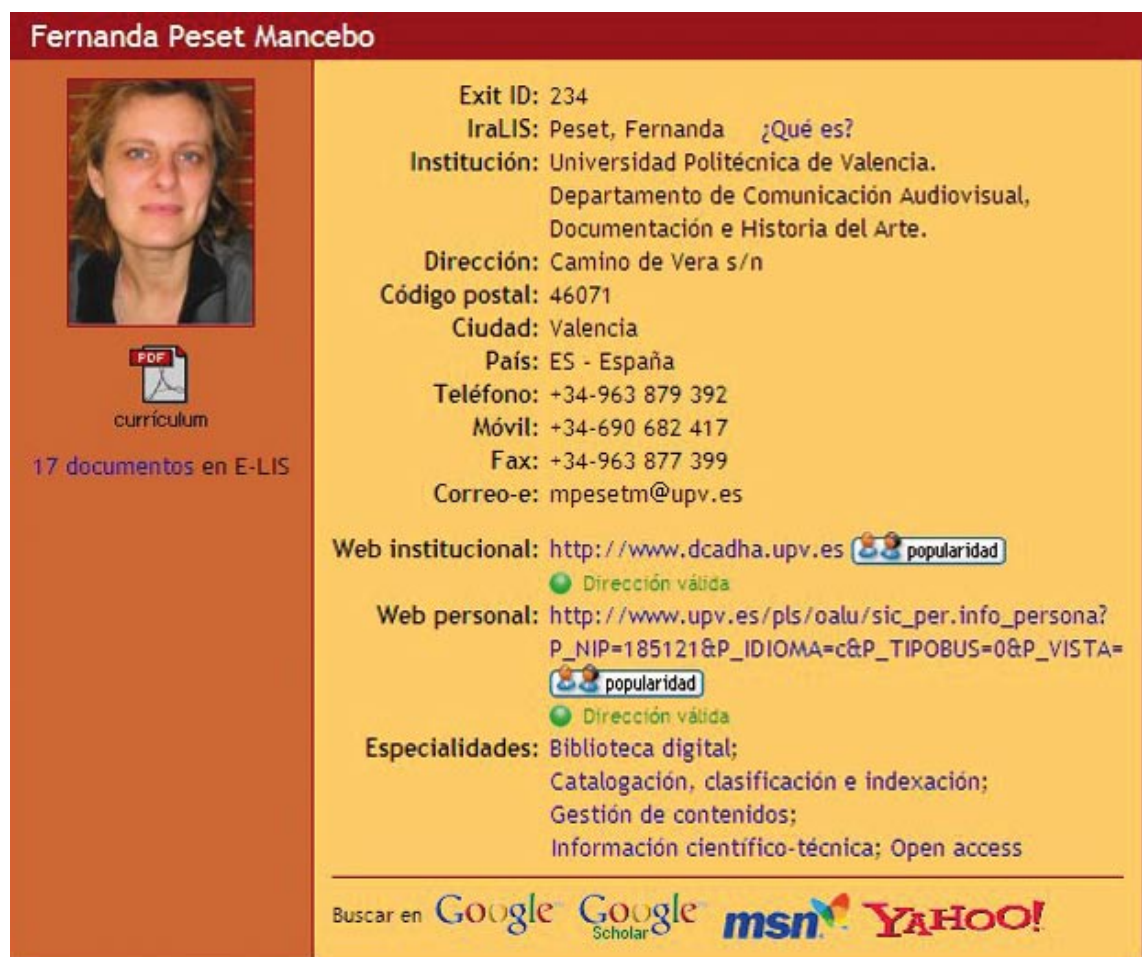

Fig. 3. Ejemplo de ficha

aparición de nuevos autores: observatorio de la profesión.

- Acceso a la publicación científica de ciertos autores (vía páginas personales, repositorio E-LIS, Google Scholar, etc.).

\subsection{Actividades de coopera- ción}

EXIT ha establecido acuerdos con los siguientes proyectos e iniciativas, con el propósito de formar un auténtico y completo sistema de información (Baiget; Peset; Subirats; Rodríguez-Gairín; Ferrer; Ontalba, 2007):

$\boldsymbol{E}$-LIS: Repositorio de acceso abierto de documentos sobre biblioteconomía y documentación.

\section{http://eprints.rclis.org/}

BiD: Revista electrónica de biblioteconomía y documentación, publicada por la Facultad de ByD de la Universidad de Barcelona.

\section{http://www.ub.es/bid/}

EPI: Revista "El profesional de la información”.

http://www.elprofesionaldelainformacion.com
Documentación digital: Máster online y diploma de postgrado a través de internet en documentación y biblioteconomía.

http://www.documentaciondigital.org/

Biblios: Revista de bibliotecología y ciencias de la información.

\section{http://www.bibliosperu.com/}

IraLIS: International registry for authors in library and information science.

\section{http://iralis.org}

\subsection{Evolución futura}

Cualquier producto debe mejorar siempre y prever circunstancias futuras para poder acoplarse a los tiempos que le toque vivir. Es por ello que las diversas tendencias, tanto sociales como técnicas (bibliotecas digitales, open access, microformatos), no pueden pasar desapercibidas.

Aparte de las tendencias, es vital que exista también una masa crítica de importancia. EXIT acaba de sobrepasar el millar de registros (agosto de 2007) y está creciendo todavía en una clara fase de joven expansión. Hay que tener en cuenta que el propósito inicial de sus creadores fue listar los principales profesionales del área geográfica latina, desde Italia hasta Chile, pasando por Portugal y Brasil - algo que, por ejemplo, se ve reflejado en la composición del actual Comité Evaluador-. Luego se incluyeron varias personas hispano hablantes de otros países, y finalmente, en febrero de 2007, y a raíz de la estrecha cooperación con E-LIS se abrió a todo el mundo.

En estos momentos se trabaja para que desde los países no-hispanos todas las pantallas de EXIT aparezcan en inglés; a continuación se ampliará el Comité, y finalmente se anunciará el nuevo enfoque por las listas profesionales de habla inglesa.

El hecho de fijarse un techo de 5.000 fichas para un futuro cercano, en vez de las mil actuales, obliga a replantearse el sistema de búsqueda interno. Ello pasa por:

- Ampliar la lista de descriptores controlados en base a los actuales descriptores libres, que se tomaran como sugerencias.

- Poder cruzar descriptores.

- Añadir el campo "Idiomas hablados"

- Relacionar los miembros del directorio con grupos o colectivos profesionales.

Como es lógico, la normalización de nombres de autores y de instituciones es un aspecto muy importante para EXIT. En este sentido se ha implementado un enlace automático con el ya citado International Registry of Authors in Library and Information Sciences (IraLIS) y se está pendiente de las decisiones de la Fundación Española para la Ciencia y la Tecnología (Fecyt) para adoptar la normativa que se apruebe respecto la normalización 
del nombre de las instituciones. Evidentemente, el carácter internacional de EXIT también obliga a sus gestores a seguir las iniciativas de control de autoridades que se publiquen en otros países.

Para facilitar su conectividad con otros sistemas se podrá difundir la información del directorio por medio de protocolos RSS -para alertar sobre nuevos miembros-, $\mathrm{u}$ OAI-PMH que permita, por ejemplo, crear listas de sugerencias de nombres en bases de datos.

\section{Resultados y discusión}

\subsection{Distribución geográfica de inscritos}

A continuación se muestra la distribución geográfica de los inscritos para aquellos países con un mínimo de 10:

Como se puede observar, el predominio español en el directorio sigue siendo importante con el $66 \%$ de los usuarios, aunque se destaca la creciente presencia suramericana: Argentina, Brasil, Colombia, Chile y México superan los 20 registros. El 8\% de los registros corresponden a países con menos de 10 registros.

En cuanto a países europeos, Italia y Reino Unido (17 y 12 registrados respectivamente) son los más representados. Estados Unidos presenta 13 registros.

Se destaca la ausencia de países con tradición y potencia investigadora como puedan ser Francia y Alemania. La presencia de otros continentes es prácticamente testimonial.

Tal y como se ha dicho antes, el próximo lanzamiento de EXIT por las listas de discusión de habla inglesa hará cambiar radicalmente estas cantidades.

\subsection{Análisis y comparación de los descriptores}

Se procede ahora a analizar los descriptores desde dos puntos de vista diferentes; por una parte la

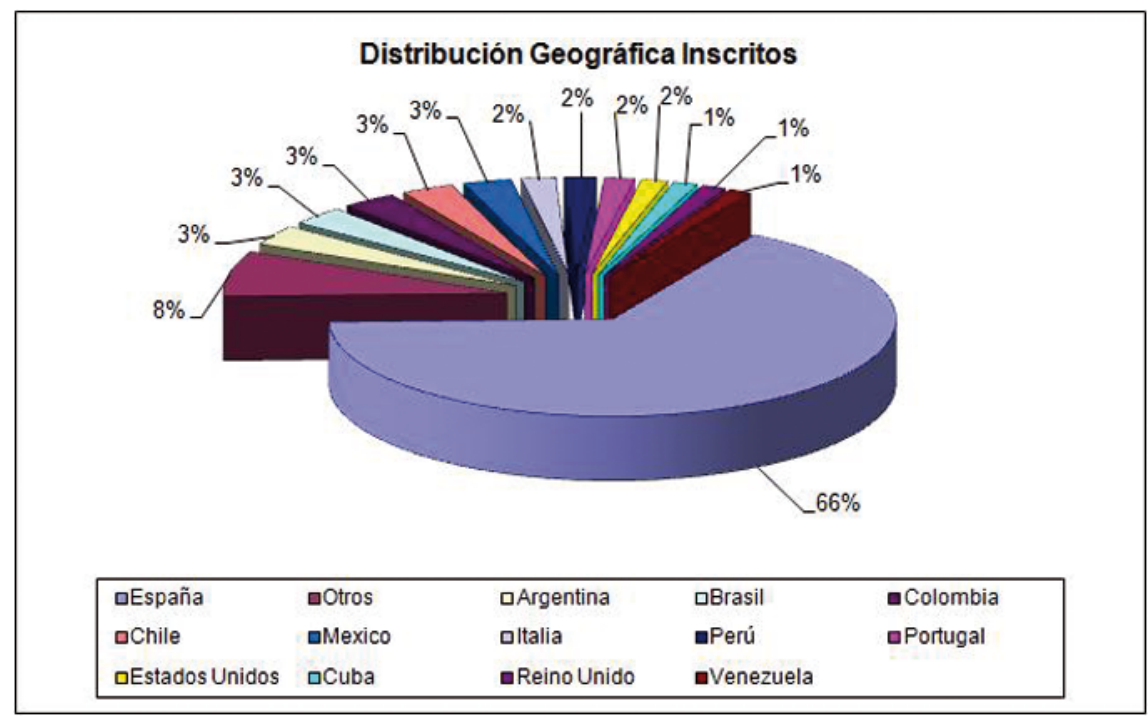

Fig. 4. Distribución geográfica (países con más de 10 registros)

distribución de los descriptores usados por los profesionales cuando se registran a través del formulario (sin tener en cuenta los descriptores libres) y, por otra parte, los descriptores más usados en la interfaz de búsqueda.

La siguiente gráfica muestra la distribución de los descriptores usados durante el registro:

Se observa cómo su envolvente es prácticamente igual a la obtenida por Hassan y Herrero, pese a que algunos descriptores han cambiado de posición.

El descriptor más usado es "Gestión de la información y del conocimiento", un descriptor demasiado general (es como si en un directorio de matemáticas, los usuarios pusiesen como materia que dominan las matemáticas, es decir, esto no nos aportaría mucha información). La "recuperación de información” y las "bibliotecas digitales" ocupan respectivamente el segundo y tercer puesto.

Respecto al trabajo de Hassan y Herrero, destaca el gran aumento de los términos "información científica-técnica" y "open access", debido a los importantes eventos internacionales que han tenido lugar recientemente y que se han reflejado en el directorio.
Destaca también la poca presencia de descriptores de disciplinas relacionadas con el mundo de los archivos, sin duda debida a la poca presencia de estos profesionales en el directorio. El primer descriptor es “archivos administrativos”, que ocupa el puesto $\mathrm{n}^{\mathrm{o}} 31$.

Otros datos de interés son el alto puesto alcanzado por el descriptor "información biomédica" (puesto 14) y los relativamente bajos puestos que obtienen los descriptores relacionados con las tareas relacionadas con el tratamiento documental (clasificación, indización, catalogación, etc.).

A continuación se muestra la distribución de los descriptores usados en las búsquedas:

Describimos algunas de las diferencias observadas en las posiciones de los descriptores:

- Los tres primeros descriptores son los mismos pero cambiados de posición, ocupando el primer lugar "biblioteca digital". "Gestión de la información y conocimiento" y "recuperación de información" ocupan el segundo y tercer lugar respectivamente.

- Puesto elevado (octavo) para "bibliometría y cibermetría", descriptor que ocupaba el puesto 24 en descriptores usados por los registrados. 


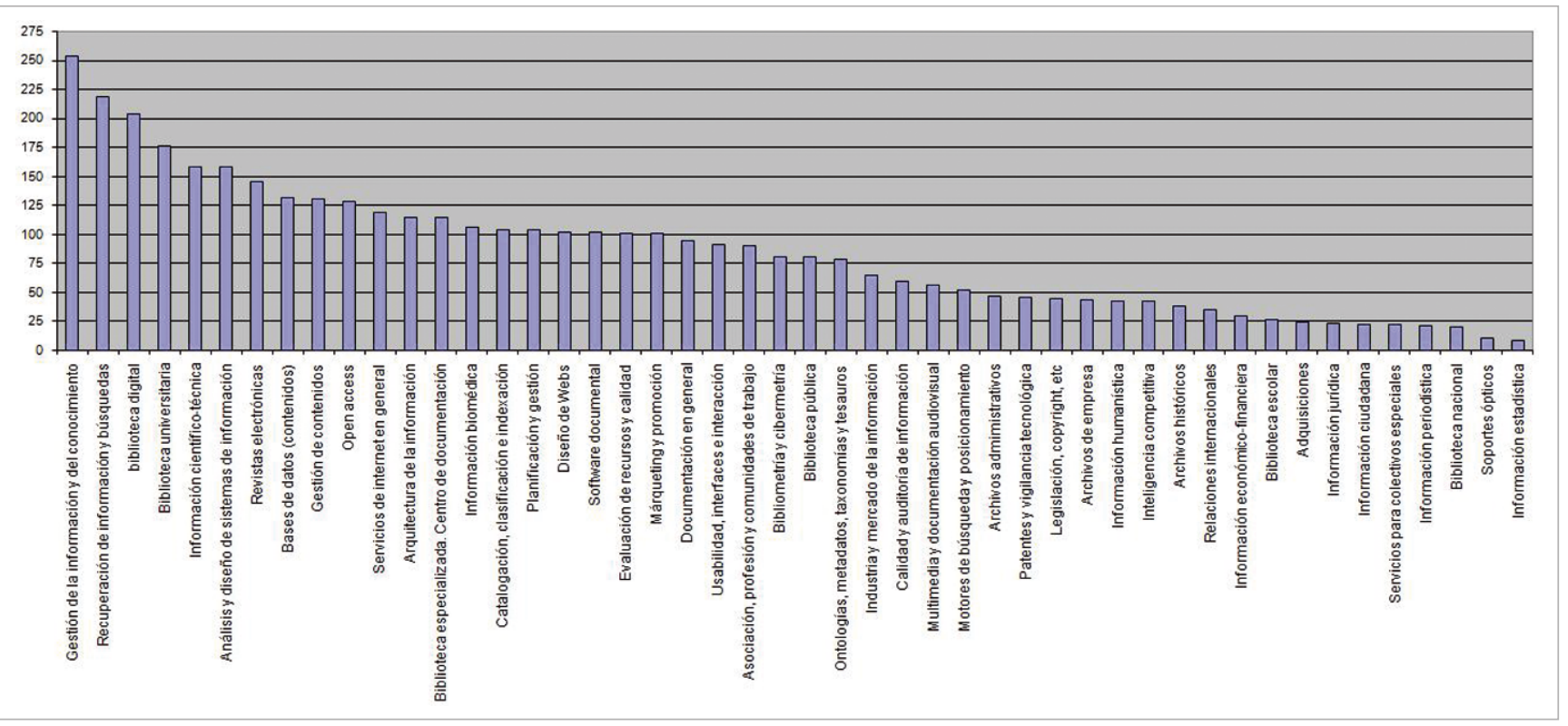

Fig. 5. Descriptores usados en la inscripción

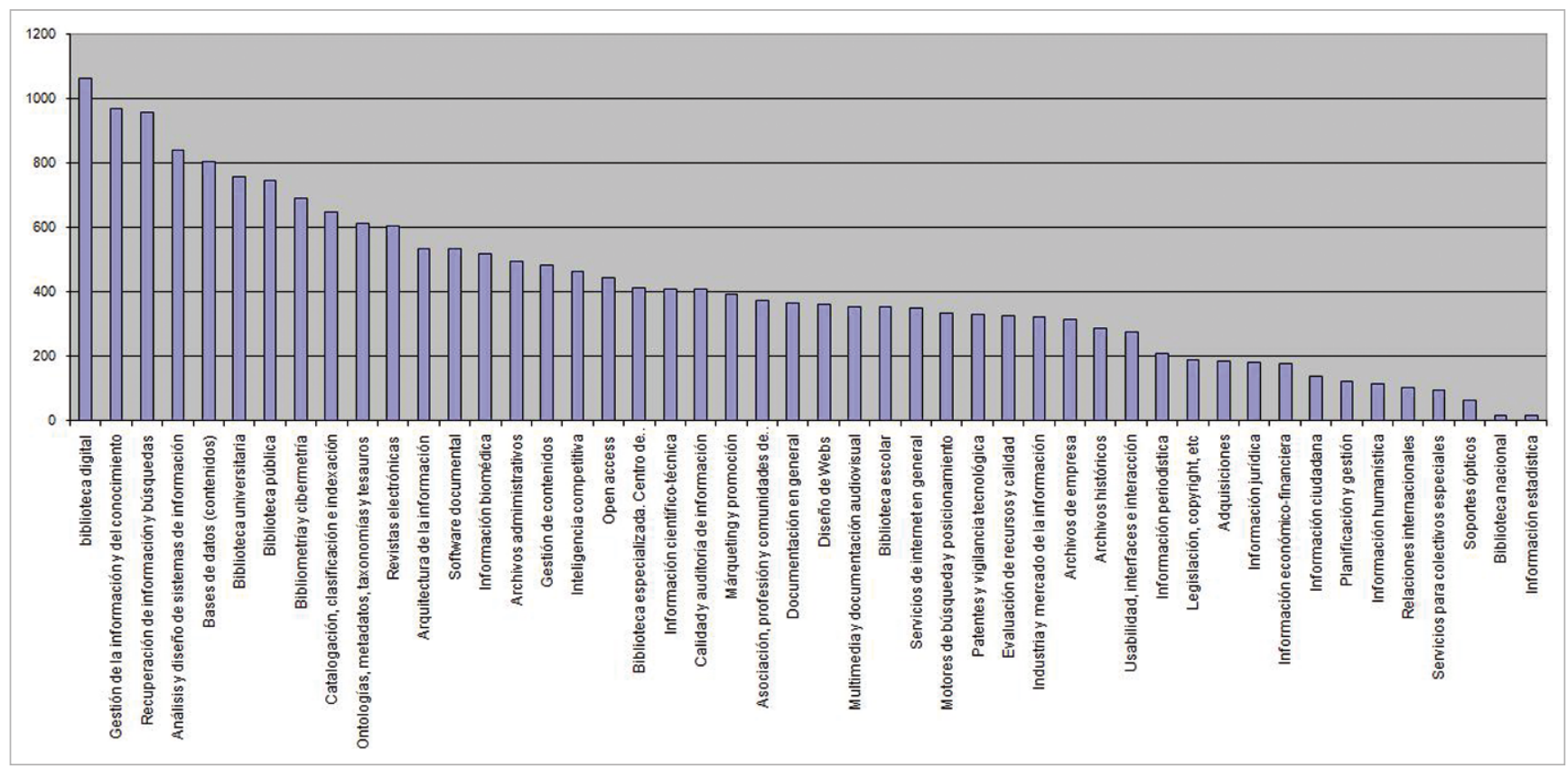

Fig. 6. Descriptores usados por los usuarios en búsquedas

- “información biomédica” vuelve a aparecer en el puesto 14 , relativamente alto, cuando existen otros descriptores relacionados con fuentes de información que aparecen en los últimos puestos (por ejemplo los relacionados con la información económica-financiera y con las fuentes de información estadística).

- En cuanto a bibliotecas, la "biblioteca universitaria” es la primera en aparecer, lo cual también podría tener cierta relación con el alto valor del descriptor "información científica-técnica”.

- Los descriptores "biblioteca digital” y “open access” están muy separados, cuando deberían ofrecer, en teoría, resultados más parecidos, como así ocurría en la anterior gráfica.

\subsection{Visitas}

En primer lugar se muestra una comparativa entre los clics recibidos por EXIT en su primer mes de funcionamiento (junio 2005) y los recibidos en junio de 2007:

Se observa un pico importante en junio de 2005, correspondiente a los días posteriores al anuncio de la aparición del directorio en algunas listas de distribución. Por lo demás se observa un ligero aumento del número de clics en los dos últimos años, además de ofrecer una envolvente menos pronunciada en los valles generados por los fines de semana, donde el acceso disminuye. 


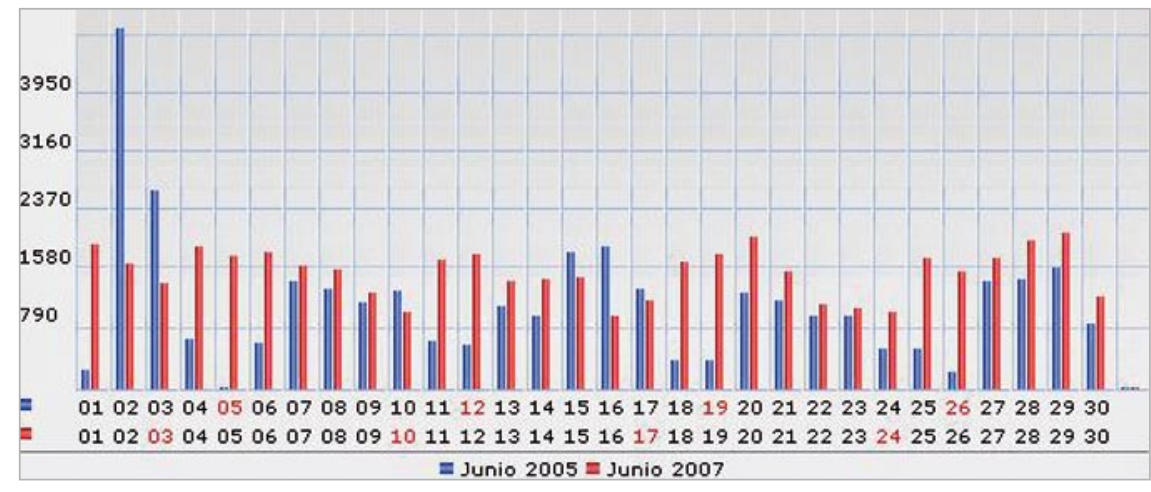

Fig. 7. Comparación de clics recibidos entre junio de 2005 y junio de 2007

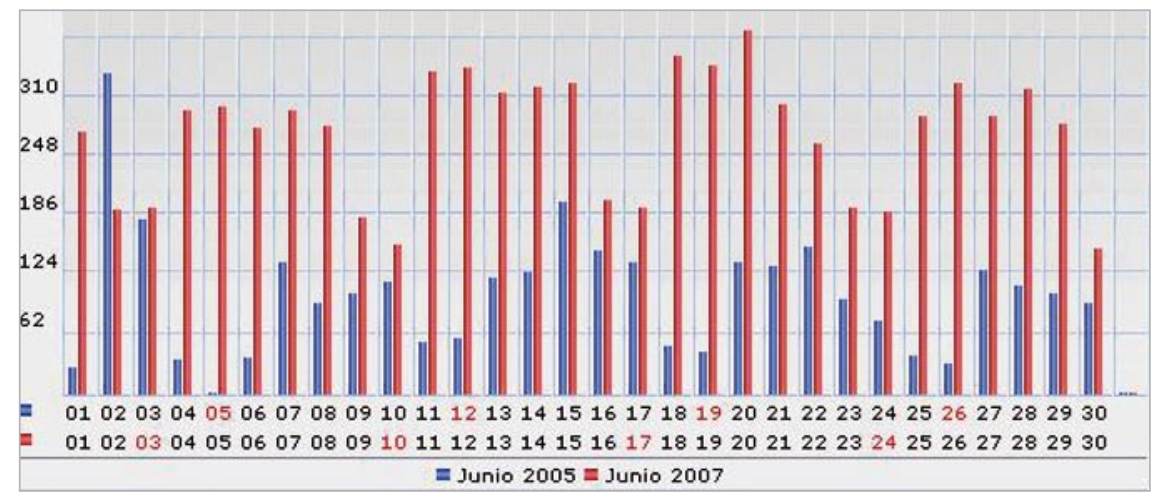

Fig. 8. Comparación de visitantes diferentes recibidos entre junio de 2005 y junio de 2007 en las gráficas correspondientes al mes de junio, mientras que el segundo es menos acusado.

\subsection{Acceso al directorio}

A continuación se muestra en detalle el modo de acceso al directorio durante el último año.

\section{Por navegador}

Cerca del $80 \%$ de los accesos provienen de Internet Explorer, mientras que el siguiente navegador más usado es Firefox a mucha distancia del anterior $(18,9 \%)$. La gráfica completa y el número de visitas por navegador se muestran seguidamente:

\section{Por fuentes de tráfico}

Las fuentes de tráfico nos proporcionan información acerca de los lugares desde los cuales se ha accedido al directorio. La siguiente figura detalla este aspecto:
En cuanto al número de visitantes, tomando en consideración la misma comparativa de meses, se obtienen los siguientes resultados:

En este caso las diferencias son más notables, observándose un crecimiento ligeramente mayor del doble de visitantes respecto al primer mes de funcionamiento.

Centrándonos en las visitas, en este caso totales, recibidas durante 2007, resulta interesante focalizar la atención en los meses de marzo y abril, meses en los cuales el directorio estableció un contacto vía correo electrónico con los profesores universitarios con docencia en facultades de Biblioteconomía y Documentación de universidades españolas. El contacto se estableció en dos fases (12 de marzo y 23 de abril). Las gráficas de visitas se pueden observar a continuación:

El primer contacto con los profesores se ve claramente reflejado

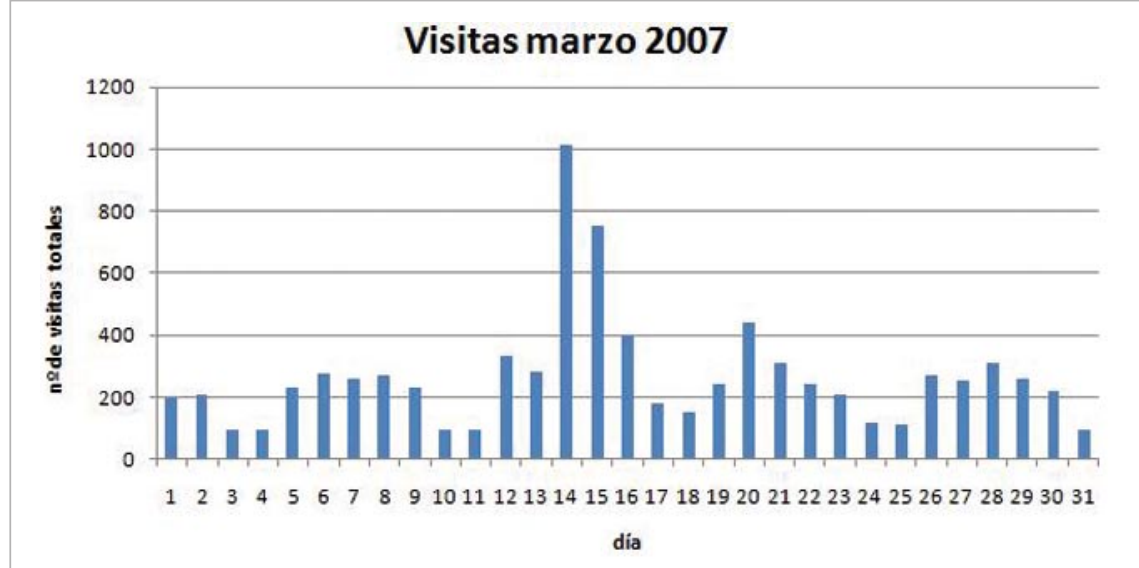

Visitas abril 2007

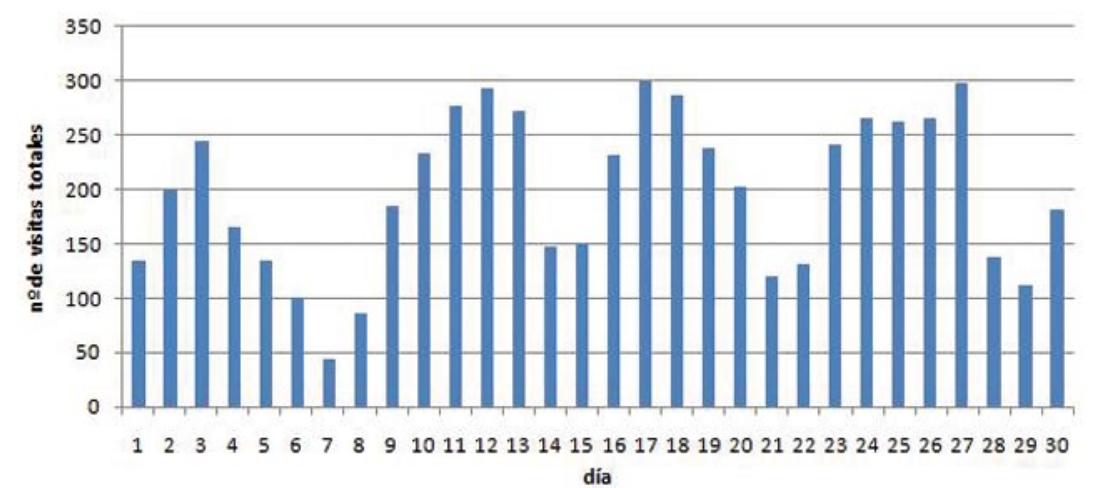

Fig. 9. Visitas totales recibidas durante los meses de marzo y abril de 2007. Fuente: Google Analytics 
La gran diferencia observada entre el acceso mediante motores de búsqueda y sitios web es indicativa de que el usuario llega al directorio tras realizar una búsqueda en algún motor concreto sin tener por qué tener en mente EXIT.

De hecho se ha constatado un alto porcentaje de visitas desde $G o$ ogle Imágenes, lo que indica que muchos usuarios han buscado probablemente una fotografía o algún dato concreto de algún profesional y han recuperado la ficha EXIT, lo cual es posible gracias al excelente posicionamiento que tienen éstas en Google.

Las visitas registradas por motor de búsqueda se muestran seguidamente:

Por el contrario, el bajo nivel alcanzado por los accesos desde sitios web de referencia, puede indicar que el directorio no está siendo enlazado desde otros sitios web de la profesión, como podrían ser de bibliotecas o de recursos de información.

En la siguiente figura se detallan los diez sitios web de referencia desde los cuales más se visita al directorio:

Es muy de destacar que el tercer puesto recaiga en $E$-LIS, uno de los mayores repositorios de nuestra profesión. Ello nos indica que los usuarios, una vez han realizado algún tipo de búsqueda en E-LIS, desean conocer más sobre el autor y acceden a EXIT gracias a la cooperación entre ambos sistemas. Resulta extraño que no aparezcan bibliotecas españolas entre los primeros puestos. La primera biblioteca que aparece (en séptimo lugar) es la de la Pontificia Universidad Católica de Valparaíso, de Chile. Este análisis indica que los gestores de EXIT deben dirigirse a los webmasters o a los responsables de bibliotecas pidiéndoles que en sus repertorios de recursos incluyan EXIT.

\begin{tabular}{|lrr|}
\hline Navegador & Visitas & $\begin{array}{r}\text { Porcentaje de } \\
\text { visitas }\end{array}$ \\
\hline Internet Explorer & 42.710 & $78,34 \%$ \\
\hline Firefox & 10.308 & $18,91 \%$ \\
\hline Safari & 481 & $0,88 \%$ \\
\hline Opera & 434 & $0,80 \%$ \\
\hline Mozilla & 350 & $0,64 \%$ \\
\hline
\end{tabular}

Fig. 10. Porcentaje de visitas por navegador web. Fuente: Google Analytics

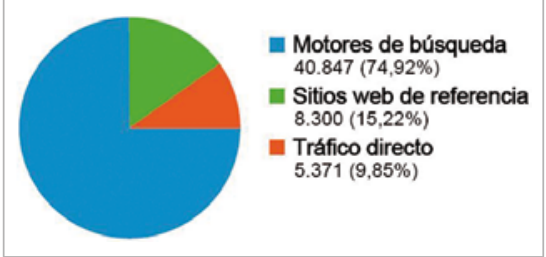

Fig. 11. Porcentaje de fuentes de tráfico. Fuente: Google Analytics

\begin{tabular}{|l|r|r|r|r|r|}
\hline Fuente & Visitas & Páginas/visita & $\begin{array}{c}\text { Promedio de } \\
\text { tiempo en el } \\
\text { sitio }\end{array}$ & $\begin{array}{c}\text { Porcentaje de } \\
\text { visitas nuevas }\end{array}$ & $\begin{array}{c}\text { Porcentaje de } \\
\text { abandonos }\end{array}$ \\
\hline google & 39.336 & 3,42 & $00: 01: 41$ & $78,93 \%$ & $69,87 \%$ \\
\hline msn & 791 & 1,97 & $00: 00: 42$ & $94,31 \%$ & $75,98 \%$ \\
\hline yahoo & 528 & 4,78 & $00: 01: 53$ & $81,25 \%$ & $67,61 \%$ \\
\hline search & 128 & 2,91 & $00: 01: 06$ & $97,66 \%$ & $67,19 \%$ \\
\hline altavista & 28 & 3,50 & $00: 00: 23$ & $85,71 \%$ & $82,14 \%$ \\
\hline aol & 21 & 1,76 & $00: 02: 37$ & $80,95 \%$ & $71,43 \%$ \\
\hline alltheweb & 4 & 1,25 & $00: 00: 06$ & $100,00 \%$ & $75,00 \%$ \\
\hline seznam & 3 & 3,67 & $00: 00: 13$ & $100,00 \%$ & $66,67 \%$ \\
\hline alice & 3 & 1,00 & $00: 00: 00$ & $100,00 \%$ & $100,00 \%$ \\
\hline lycos & 3 & 1,00 & $00: 00: 00$ & $100,00 \%$ & $100,00 \%$ \\
\hline
\end{tabular}

Fig. 12. Número de visitas por motor de búsqueda. Fuente: Google Analytics

\begin{tabular}{|l|r|r|r|r|r|}
\hline Fuente & Visitas & Páginas/visita & $\begin{array}{c}\text { Promedio de } \\
\text { tiempo en el } \\
\text { sitio }\end{array}$ & $\begin{array}{c}\text { Porcentaje de } \\
\text { visitas nuevas }\end{array}$ & $\begin{array}{c}\text { Porcentaje de } \\
\text { abandonos }\end{array}$ \\
\hline directorioexit.info & 1.995 & 8,11 & $00: 04: 52$ & $49,82 \%$ & $32,03 \%$ \\
\hline google.es & 463 & 3,46 & $00: 01: 38$ & $82,94 \%$ & $66,74 \%$ \\
\hline eprints.rclis.org & 298 & 5,75 & $00: 03: 25$ & $58,39 \%$ & $39,93 \%$ \\
\hline thinkepi.net & 293 & 6,19 & $00: 03: 30$ & $67,24 \%$ & $36,18 \%$ \\
\hline bitacoradeunbibliotecario.blogspot.com & 283 & 2,08 & $00: 01: 09$ & $88,69 \%$ & $76,68 \%$ \\
\hline elprofesionaldelainformacion.com & 279 & 3,25 & $00: 02: 36$ & $45,88 \%$ & $41,94 \%$ \\
\hline biblioteca.ucv.cl & 200 & 2,48 & $00: 00: 50$ & $93,00 \%$ & $75,50 \%$ \\
\hline iralis.org & 163 & 5,50 & $00: 02: 56$ & $41,72 \%$ & $35,58 \%$ \\
\hline lacoctelera.com & 113 & 3,91 & $00: 02: 16$ & $59,29 \%$ & $64,60 \%$ \\
\hline catorze.com & 106 & 4,80 & $00: 02: 09$ & $66,04 \%$ & $63,21 \%$ \\
\hline
\end{tabular}

Fig. 13. Número de visitas desde sitios web de referencia. Fuente: Google Analytics

La primera biblioteca española aparece en el puesto número 13 (Universidad de Granada) y sorprendentemente, en el puesto 19, la Red de bibliotecas municipales de San Javier (Murcia).

Otro aspecto revelador es la alta presencia de la biblioblogsfera (blogs sobre Biblioteconomía, Documentación, Archivística y afines) entre los primeros puestos. En concreto se han detectado ocho blogs (prácticamente los más importantes) entre los 25 primeros lugares de acceso.
Por último, se advierte la ausencia de accesos desde las diversas asociaciones nacionales (únicamente aparece Fesabid) entre las 25 primeras webs de referencia.

\section{Por lugar de acceso}

Europa es el lugar desde donde más se ha accedido. El continente americano presenta unos niveles aceptables, sin duda por la creciente presencia de autores de dichos lugares de procedencia. El acceso desde el resto de continentes es bajo en la actualidad: 
En cuanto a España, a continuación se muestra un detalle del número de visitas por ciudad:

Madrid es la ciudad, con diferencia, desde donde aparentemente más se ha accedido a EXIT, y Barcelona se encuentra a bastante distancia, a pesar de que en el momento de escribir esto hay 168 inscritos de Barcelona y 127 de Madrid. Esta anomalía de los datos de Google Analytics se debe a que las universidades y las instituciones públicas catalanas han cambiado sus dominios ".es" por otros genéricos como ".edu", “.net”, “.org” y recientemente ".cat" (este último tampoco es un dominio geográfico sino cultural).

Después de esas dos ciudades aparecen Sevilla y Valencia.

Las visitas recibidas desde los dominios genéricos, y por tanto sin localización geográfica inmediata, son casi un $40 \%$ del total.

Fuera de España, el segundo gran foco de accesos proviene desde Latinoamérica. A continuación podemos observar la distribución de accesos por país:

Finalmente, se muestran los datos correspondientes a Estados Unidos, donde se observa cómo el estado de California es el más activo. También se debe hacer constar que aparece un alto porcentaje de accesos sin localizar (cerca del 20\%).

\section{Conclusiones}

Tras dos años en funcionamiento, el directorio EXIT se encuentra en fase de expansión de cobertura, lo que está provocando un crecimiento de registros tal que lo han convertido en el directorio español gratuito en internet de mayor tamaño. Y ello a pesar de que sólo pueden inscribirse profesionales que cuenten ya con experiencia. La gran visibilidad de las fichas en Google es sin duda uno de sus mayores alicientes.

\begin{tabular}{|l|r|r|r|r|r|}
\hline Continente & Visitas & Páginas/visita & $\begin{array}{c}\text { Promedio de } \\
\text { tiempo en el } \\
\text { sitio }\end{array}$ & $\begin{array}{c}\text { Porcentaje de } \\
\text { visitas nuevas }\end{array}$ & $\begin{array}{c}\text { Porcentaje de } \\
\text { abandonos }\end{array}$ \\
\hline Europe & 40.296 & 4,37 & $00: 02: 06$ & $73,46 \%$ & $62,61 \%$ \\
\hline Americas & 13.859 & 2,68 & $00: 01: 43$ & $86,12 \%$ & $71,53 \%$ \\
\hline Asia & 234 & 3,49 & $00: 03: 06$ & $81,20 \%$ & $67,95 \%$ \\
\hline Africa & 76 & 5,66 & $00: 03: 18$ & $77,63 \%$ & $69,74 \%$ \\
\hline (not set) & 29 & 2,55 & $00: 03: 22$ & $75,86 \%$ & $75,86 \%$ \\
\hline Oceania & 24 & 1,54 & $00: 00: 21$ & $79,17 \%$ & $79,17 \%$ \\
\hline
\end{tabular}

Fig. 14. Porcentaje de visitas por continente. Fuente: Google Analytics

\begin{tabular}{|l|r|r|r|}
\hline Cludad & Visitas & Visitas & \multicolumn{2}{|c|}{ Páginas/visita } \\
\hline (not set) & 14.374 & $39,24 \%$ & \\
\hline Madrid & 11.564 & $31,57 \%$ & \\
\hline Barcelona & 4.786 & $13,06 \%$ & $31,57 \%$ \\
\hline Sevilla & 981 & $2,68 \%$ & \\
\hline Valencia & 949 & $2,59 \%$ & \\
\hline Salamanca & 383 & $1,05 \%$ & \\
\hline Santiago de Compostela & 351 & $0,96 \%$ & \\
\hline Bilbao & 344 & $0,94 \%$ & \\
\hline La Coruna & 317 & $0,87 \%$ & \\
\hline Zaragoza & 299 & $0,82 \%$ & \\
\hline
\end{tabular}

Fig. 15. Porcentaje de visitas por ciudad. Fuente: Google Analytics

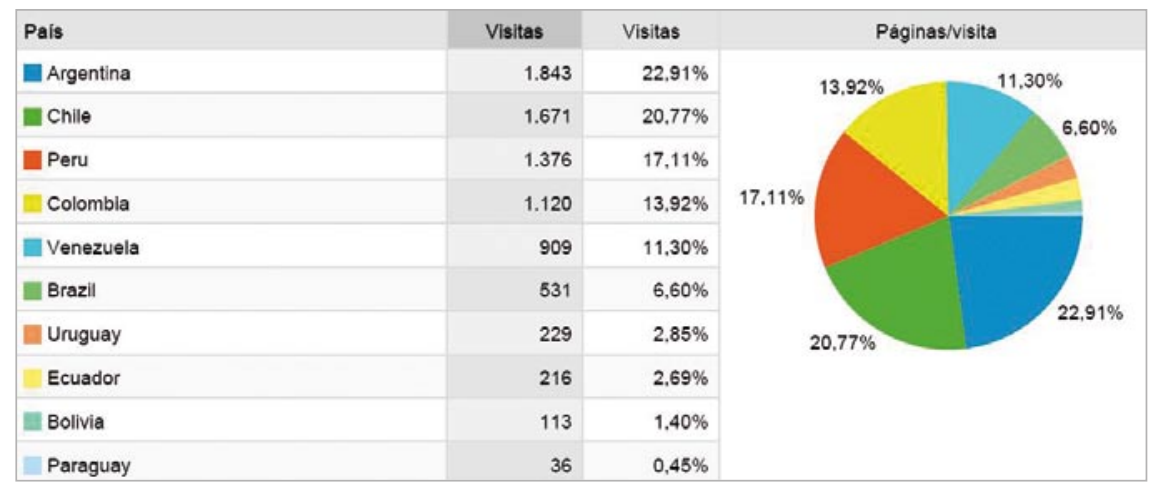

Fig. 16. Porcentaje de visitas por país (Latinoamérica). Fuente: Google Analytics

\begin{tabular}{|c|c|c|c|}
\hline Región & Visitas & Visitas & Páginas/visita \\
\hline California & 220 & $20,66 \%$ & \multirow{10}{*}{$8,08 \%$} \\
\hline (not set) & 204 & $19,15 \%$ & \\
\hline Florida & 89 & $8,36 \%$ & \\
\hline Texas & 86 & $8,08 \%$ & \\
\hline New York & 73 & $6,85 \%$ & \\
\hline New Jersey & 40 & $3,76 \%$ & \\
\hline En Illinois & 39 & $3,66 \%$ & \\
\hline Pennsylvania & 31 & $2,91 \%$ & \\
\hline In Alabama & 27 & $2,54 \%$ & \\
\hline Massachusetts & 26 & $2,44 \%$ & \\
\hline
\end{tabular}

Fig. 17. Porcentaje de visitas por país (Estados Unidos). Fuente: Google Analytics

La cobertura de inscritos está todavía dominada por españoles, aunque los porcentajes están comenzando a nivelarse.
El análisis de descriptores muestra que los tres términos más usados por los profesionales a la hora de registrarse, son también los 


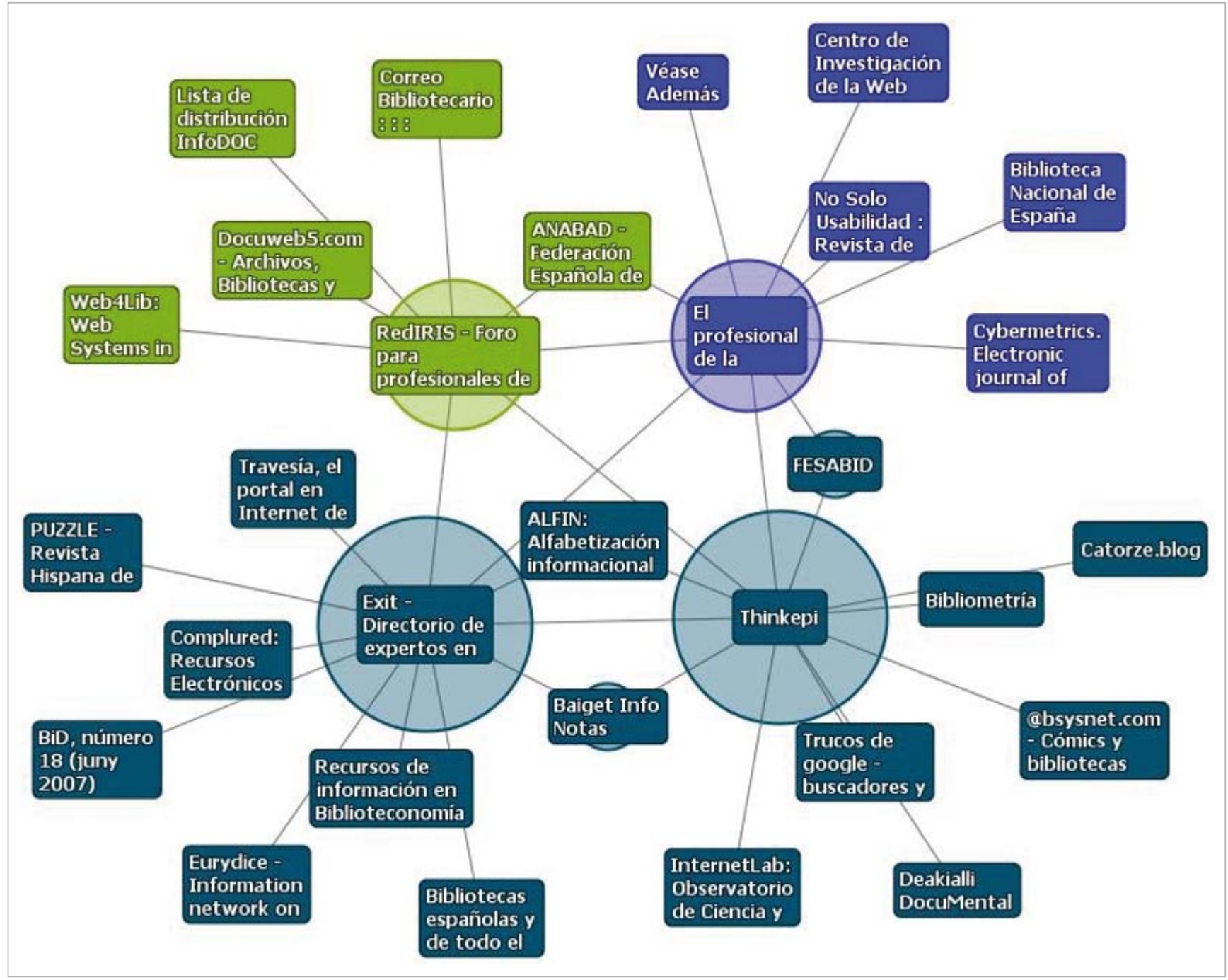

Fig. 18. Mapa TouchGraph de EXIT, mostrando su entorno en la Red

mismos más usados en las búsquedas. El descriptor "Gestión de la información y del conocimiento" es demasiado general y es usado mayormente como término comodín. Los otros dos ("Bibliotecas digitales" y "Recuperación de información y búsquedas"), se evidencian como las áreas que más interesan a los profesionales que acceden al directorio. Áreas afines a las bibliotecas digitales como son "Open access" y "Revistas electrónicas", aparecen también en los primeros puestos, con una clara tendencia a crecer.

Sin tener en cuenta los tres descriptores más usados, existen ciertas diferencias, entre las que destaca "bibliometría y cibermetría", apenas usado por los registrados $\mathrm{y}$, en cambio, muy buscado por los usuarios.

Las fuentes de información de carácter técnico ("Información científica-técnica", "Información biomédica") aparecen con frecuencia, mientras que los descriptores de fuentes de información más humanística son muy poco frecuentes, al igual que los de información económica, financiera y estadística, que prácticamente son nulos.

En relación con los sitios desde donde los usuarios acceden al directorio, destaca la alta presencia de los blogs de Biblioteconomía y Documentación y la baja presencia de asociaciones profesionales y bibliotecas.

Las cifras de América Latina, tanto en profesionales registrados como en número de visitas, hacen patente que el directorio ha sido bien recibido allí y está siendo usado como fuente de información.

Se considera importante el creciente porcentaje de visitas desde Estados Unidos, donde se observa al estado de California como el más activo, con la reserva de que existe un alto porcentaje de accesos sin identificar.

\section{Bibliografía}

1. Aleixandre, Rafael. ¿Quién es quien es documentación médica en España? Consultado en: 12-07-07.

http://www.uv.es/ docmed/quienes/quien.html

2. Baiget, Tomàs. "Nuevos formatos del Directorio IWE". En: Information world en español, 1995, n. 35, junio, p. 14.

http://www.elprofesionaldelainformacion.com/ contenidos/1995/junio/nuevos_formatos_del_directorio_iwe.html 
3. Baiget, Tomàs; Peset, Fernanda; Subirats, Imma; Rodríguez-Gairín, Josep-Manuel; Ferrer, Antonia; Ontalba-Ruipérez, José-Antonio. "Authority records for author's names in Library and Information Science". En: Proceedings 5th Workshop on Innovations in Scholarly Communication (OAI5), Geneva, Switzerland: CERN, 2007. Consultado en: 11-08-07. http://eprints.rclis.org/archive/00009601/

4. Carrizo-Sainero, Gloria. Manual de fuentes de información. Zaragoza: Cegal, 2000. ISBN 84-923722-2-2

5. Ferrer, Antonia; Peset, Fernanda. "Análisis de los directorios privados de información empresarial española”. En: El profesional de la información, 2007, mayo-junio, v. 16, n. 3, pp 243-257.

6. Hassan-Montero, Yusef; Herrero-Solana, Víctor. "Visualizing library and information science from the practitioner's perpective". En:
Poster presentation submitted to the $11^{\text {th }}$ International conference of the International Society for Scientometrics and Informetrics, June 25-27, 2007. Consultado en: 12-07-07.

http://www.nosolousabilidad.com/hassan/Visualizing_LIS.pdf

7. Membrillo-Marco, Dolores, coord; Jiménez-Aleixandre, Miguel, coord. Quién es quien en información, documentación y bibliotecas. Madrid: EBSCO Information Services, 1998.

8. Rodríguez-Gairín, Josep-Manuel. "DirIWE: directorio de correo-e de los profesionales de habla hispana". En: El profesional de la información, 1998, v. 7, n. 6, junio, pp. 29-30.

http://www.elprofesionaldelainformacion.com/ contenidos/1998/junio/diriwe_directorio_de correoe_de_los_profesionales_de_habla_hispana.html

9. Rodríguez-Gairín, Josep-Manuel; Baiget, Tomàs. Directorio EXIT: directorio de expertos en el tratamiento de la información. Consultado en: $12-07-07$

http://www.directorioexit.info

Enrique Orduña-Malea, Universidad Politécnica de Valencia.

enorma@fiv.upv.es

Josep-Manuel Rodríguez-Gairín, Facultat de Biblioteconomia i Documentació, Universitat de Barcelona.

rodriguez.gairin@ub.edu

Tomàs Baiget, Institut d'Estadística de Catalunya (Idescat)

baiget@sarenet.es

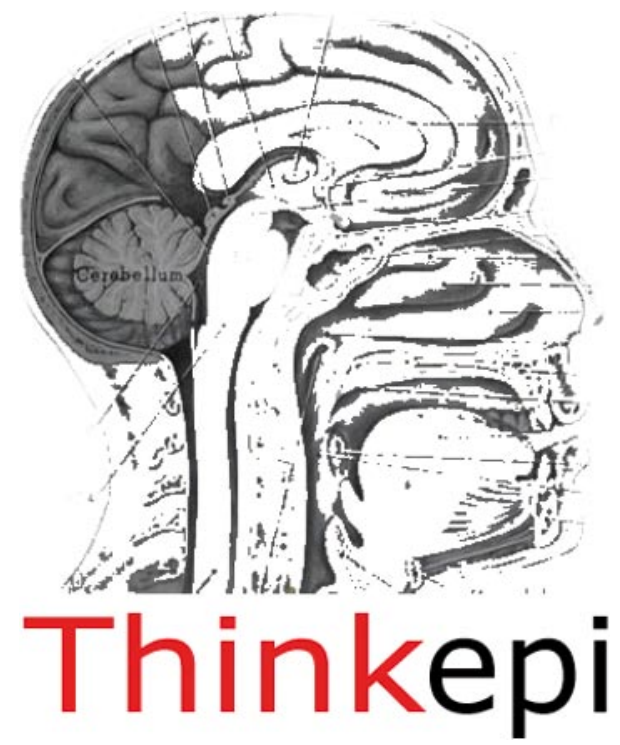

El Grupo Thinkepi está formado por 30 profesionales y académicos de la biblioteconomía y la documentación, con experiencia y reconocido prestigio, que elaboran notas con micro-estados del arte, reflexiones sobre temas profesionales de actualidad, perspectivas ya consolidadas ante nuevos productos, opiniones, observaciones, etc.

Estas notas, más una recopilación de las principales noticias e hitos del sector, se publican en los Anuarios ThinkEPI.

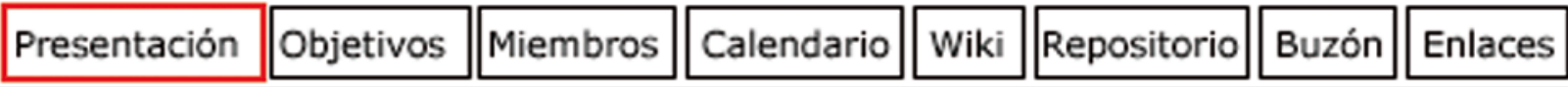

\section{ANUARIO}

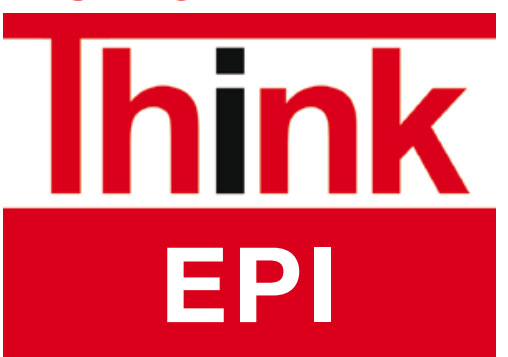

ISSN 1886-6344
Anuario ThinkEPI 2008

http://www.thinkepi.net/

\section{Estamos preparando una nueva edición del Anuario ThinkEPI}

Infórmate de los nuevos contenidos en: http://www.thinkepi.net/repositorio/

Ya puedes pasarnos tu pedido: epi@sarenet.es

Anuario ThinkEPI 2008

Anuarios ThinkEPI $2007+2008$

$89,42 €+I V A=93 €$
$115,39 €+I V A=120 €$

